\title{
Lightweight Study of Carbon Fiber Composite B-Pillar Based on Equal Stiffness Principle
}

\author{
Dongming Sun, Chao Tao \\ College of Automotive Engineering, Shanghai University of Engineering Science, Shanghai, China \\ Email: 1002508398@qq.com
}

How to cite this paper: Sun, D.M. and Tao, C. (2018) Lightweight Study of Carbon Fiber Composite B-Pillar Based on Equal Stiffness Principle. Open Access Library Journal, 5: e4822.

https://doi.org/10.4236/oalib.1104822

Received: August 2, 2018

Accepted: August 27, 2018

Published: August 30, 2018

Copyright @ 2018 by authors and Open Access Library Inc.

This work is licensed under the Creative Commons Attribution International License (CC BY 4.0).

http://creativecommons.org/licenses/by/4.0/

\section{(c) (i) Open Access}

\begin{abstract}
The replacement of carbon fiber reinforced epoxy resin matrix composite (CFRP) in the B-pillar of the body structure was discussed. Under the condition of ensuring crashworthiness, the lightweight application of B-pillar on the structural parts of the automobile was realized. Taking the steel B-pillar of a certain model as the prototype, CFRP is used for the same stiffness design, and the stiffness, crashworthiness and lightweight performance of the composite B-pillar relative to the high-strength steel B-pillar are comprehensively analyzed. The results show that under the requirements of crashworthiness, the lightweight effect of CFRP on B-pillar is obvious, which is $26.27 \%$ lower than that of high-strength steel B-pillar. Therefore, under the equal stiffness design conditions, the composite material can better realize the lightweight requirements of the automobile B-pillar.
\end{abstract}

\section{Subject Areas}

Automata, Mechanical Engineering

\section{Keywords}

B-Pillar, CFRP, Lightweight, Crashworthiness

\section{Introduction}

With the rapid development of the automotive industry, the number of cars has increased significantly. Driven by automobile safety collision and energy conservation and emission reduction regulations, reducing energy consumption and reducing pollution has become an urgent task for automobile development. Reducing the weight of automobiles is one of the effective ways to solve the above problems. The application of high-strength lightweight materials and lightweight structures has become an inevitable trend in the development of automobiles. 
Carbon fiber composites have become more and more widely used in the body due to their high specific strength and specific stiffness [1] [2] [3].

At present, the application of composite materials on the body at home and abroad still uses metal components for the entire body, and only composite materials are used on some of the components. Although the CFRP material has higher cost, its specific strength, specific modulus and other properties are the most superior among the existing structural materials, and its high strength and rigidity have become the hotspots of recent research at home and abroad. Wu Fanghe et al. [4] carried out structural design of the original steel hood, replaced with composite materials, studied the basic performance of the hood through finite element simulation and prototype experiments, and discussed the replacement of steel with carbon fiber composite parts. Feasibility of the part and test its lightweight effect. Li Bei [5] conducts research on lightweight design and optimization of composite bumper to achieve lightweight bumper and better crashworthiness. Cheng Zhang et al. [6] applied composite materials to automotive fenders and optimized the structure and layup based on the Kriging model, which reduced the weight of the optimized fenders by $43.1 \%$.

There are relatively many researches on the application of carbon fiber composite materials to automobile body parts and covers, but the research and development of composite body frame and important structural parts are being developed and explored at home and abroad. Zhang Zipeng [7] conducted energy absorption research on composite cylindrical tube samples, and replaced the front longitudinal beam of the automobile with composite materials for energy absorption characteristics research. Yang Yuwei et al. [8] simplified the design according to the performance requirements of the car body design and the material of the carbon fiber composite material. Through the CAE analysis, the carbon fiber composite material was prototyped and applied to the whole vehicle. In the research of body frame composite materials, the composite material B column is used as the typical structural part of the automobile, and the material replacement research is carried out, which has certain guiding significance for the frame body structure. As the main structural part of the car side impact, the B-pillar should be based on the collision safety of the B-pillar, and the lightweight application of the B-pillar should be studied. For the composite material B-pillar research is relatively small, Xue Jiao [9] through the simplified B-pillar structure into the I-beam structure, the corresponding performance analysis, to achieve replacement lightweight.

In this paper, a high-strength steel B-pillar of a certain model is replaced with a lightweight composite CFRP. The thickness is designed based on the principle of equal stiffness. Statics and collision performance analysis is performed on the original model, and the accuracy of the model is verified by energy analysis during the collision process. Compared with the model after material replacement, it is guaranteed that the B-pillar has the same bending stiffness in low-speed collision. This paper compares the light weight and crashworthiness of two lightweight materials, which provides a basis for the lightweight design of automotive 
B-pillars.

\section{Model Establishment and Structural Analysis of B-Pillar}

\subsection{Establishment of B-Column Finite Element Model}

The B-pillar is located between the front door and the rear door and is an important bearing member during the side collision of the car. The overall shape of the B-pillar must be curved and consistent with the shape of the body. The B-column geometry model is imported into the HyperMesh pre-processing software, and the operations such as extracting the middle surface, geometric cleaning and meshing are performed, wherein the meshing is as much as possible using quadrilateral elements, and the number of triangular elements does not exceed $5 \%$ of the total number of cells, and the mesh size is $10 \mathrm{~mm}$ and the total number of grids is 11,363 . The B-pillar is mainly connected by welding, highstrength steel DC590, material properties: elastic modulus is $210 \mathrm{Mpa}$, Poisson's ratio is 0.3 , density is $7850 \mathrm{~kg} / \mathrm{m}^{3}$. The established B-column finite element model is shown in Figure 1.

\subsection{Static Analysis of Automobile B-Pillar}

The static analysis of automobile B-pillars mainly includes the stress and strain under bending conditions, and all the translational degrees of freedom and rotational degrees of freedom at the four ends of the B-pillar are constrained. A concentrated load of $1000 \mathrm{~N}$ is applied along the $\mathrm{Y}+$ direction at the middle position of the B-pillar. The analytical model is shown in Figure 2. The displacement and stress clouds of the static analysis results are shown in Figure 3 and Figure 4. The displacement of the loading point is $0.704 \mathrm{~mm}$; the maximum stress appears on the inner plate under the B-pillar with a stress value of $102 \mathrm{MPa}$.

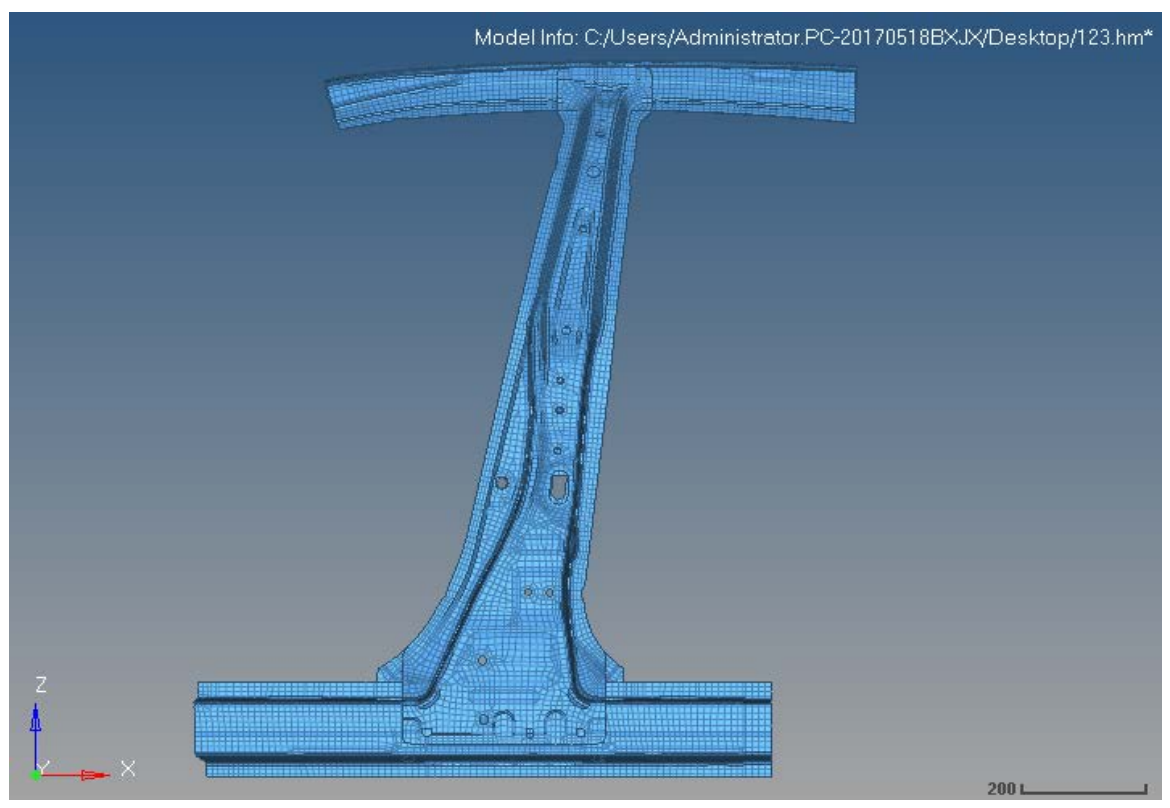

Figure 1. B-column finite element model. 


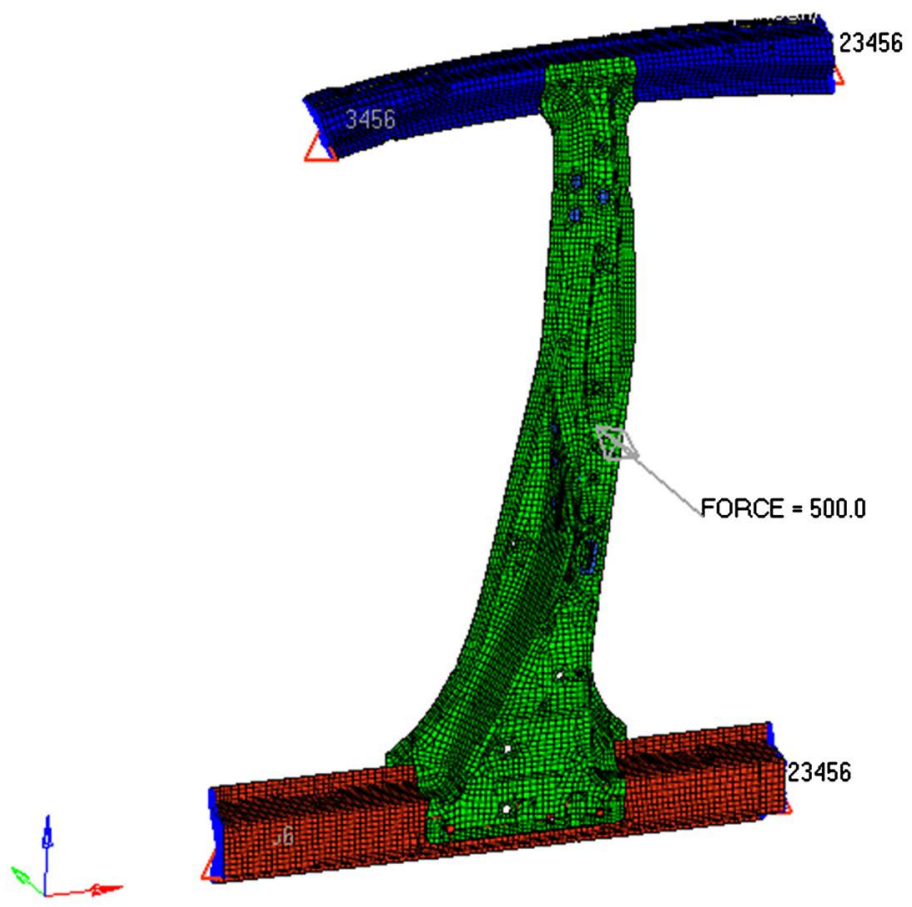

200 L

Figure 2. Analysis model.

Contour Plot

1: Model

Element Stresses (2D \& 3D)(yonMises, Mablase 1 (loadstep1) : Static Analysis : Frame 25 Analysis system

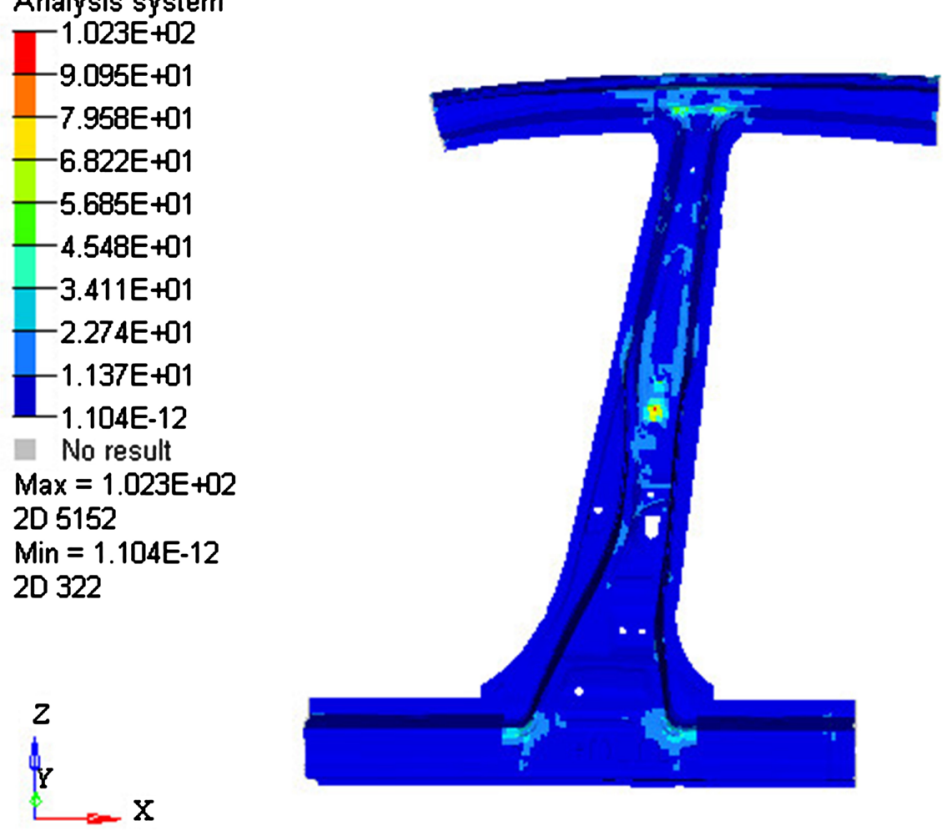

Figure 3. Displacement cloud map.

\section{Performance Analysis of CFRP Parts Replacement Model}

\subsection{Equal Stiffness Material Replacement Principle}

Based on the principle of equal stiffness replacement, the existing high-strength steel B-pillar is replaced by a carbon fiber composite material, and the B-column 
Element Stresses (2D \& 3D)(vonMises, Saktase 1 (loadstep1) : Static Analysis : Frame 25 Analysis system

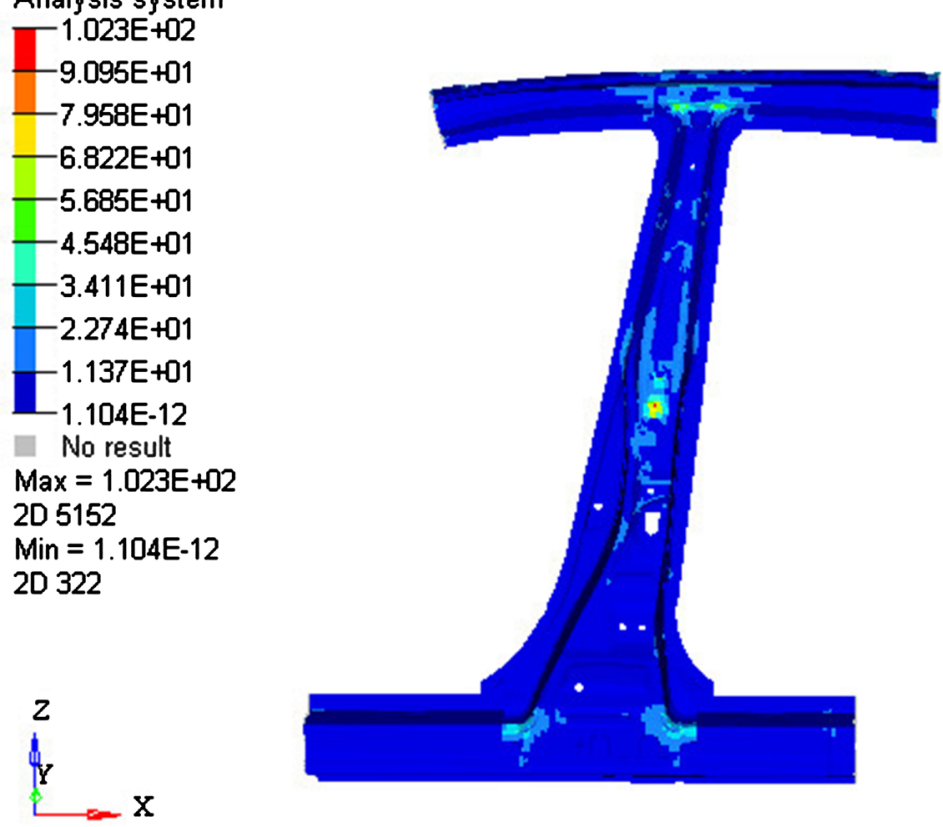

Figure 4. Stress cloud map.

lightweight design is designed to remove the reinforcing plate in the B-pillar. For the B-pillar design, three schemes are designed. The solution is to replace the inner and outer plates of the B-pillar with the composite material. The second scheme replaces the B-pillar front outer panel with the composite material. The third scheme replaces the B-pillar inner panel with the composite material, and determines the thickness of the inner and outer plates of the B-pillar through the equivalent stiffness approximation theory. among them:

$$
\begin{aligned}
E_{a} I_{a} & =E_{b} I_{b} \\
E_{a} \frac{b_{a} h_{a}{ }^{3}}{12} & =E_{b} \frac{b_{b} h_{b}{ }^{3}}{12}
\end{aligned}
$$

For thin-walled structures, the width of the cross-sectional area:

$$
b_{a}=b_{b}, \quad h_{a}=\sqrt[3]{\frac{E_{b} h_{b}{ }^{3}}{E_{a}}}
$$

In the formula: $E_{a}$ is the equivalent modulus of the laminate; $I_{a}$ is the moment of inertia of the cross section of the composite structure; $E_{b}$ is the modulus of elasticity of the original structural material; $I_{b}$ is the moment of inertia of the cross section of the original structure; $h_{a}, h_{b}$ is the cross section Height, also known as thickness.

According to the expression (c), the thickness of the replacement material can be initially determined. In order to ensure the rigidity of the structure after the replacement of the material, the thickness adjustment is often performed based on the position of the different plates. In addition, the stiffness of a single sheet metal member is a nonlinear function of thickness, and the approximate rela- 
tionship is expressed as:

$$
K=C E t^{\theta}
$$

where: $C$ is the geometric coefficient; $\mathrm{E}$ is the elastic modulus; $\mathrm{t}$ is the material thickness; $\theta$ is the thickness index coefficient. According to the expression (d), the ratio of the thickness of the material before and after the stiffness replacement:

$$
\frac{t_{1}}{t_{0}}=\left(\frac{E_{0}}{E_{1}}\right)^{\frac{1}{\theta}}
$$

where: $t_{0}$ and $t_{1}$ are the thicknesses before and after material replacement; $E_{0}$ and $E_{1}$ are the elastic moduli before and after material replacement; by expressions (d) and (e), the thickness elastic modulus of the material is equal to stiffness. Inverse ratio. For the body structure $\theta$ is usually between $1-2$.

\subsection{Design of Equal-Stiffness CFRP Material Replacement B-Pillar}

Based on the above theory, the thickness values of the inner and outer plates of the B-pillar after material replacement are shown in Table 1. The thickness index coefficients of the inner and outer plate materials are 1.81 and 2.08, respectively, which satisfy the values of $1-3$. Combining the existing technology and processing technology, three alternatives to the design of the B-pillar are proposed to study the performance of the replacement of the B-pillar. The thickness of the outer panel is shown in Figure 5.

Option 1 replace the inner and outer plates of the B-pillar with equal stiffness. The CFRP inner plate thickness is $4 \mathrm{~mm}$, the CFRP outer plate thickness is 4 $\mathrm{mm}$, the CFRP thickness is $0.25 \mathrm{~mm}$, and the inner and outer plates are 16 layers, according to $0^{\circ} / 45^{\circ} / 90^{\circ} /-45^{\circ}$ way to laminate.

Option 2 replace the outer plate of the B-pillar with equal rigidity. The thickness of the CFRP outer plate is $4.12 \mathrm{~mm}$, the thickness of each layer of CFRP is $0.25 \mathrm{~mm}$, and the inner and outer plates are laid 16 layers, paved according to $0^{\circ} / 45^{\circ} / 90^{\circ} /-45^{\circ}$. The inner plate is of the original metal structure and is rigidly connected.

Table 1. Thickness comparison before and after replacement of inner and outer plate materials.

\begin{tabular}{ccccc}
\hline Alternative scheme for B column design & Material & Equivalent elastic modulus /GPa & thickness/mm \\
\hline Basic model & Inner plate & DC590 & 210 & 1.6 \\
& Outer plate & DC590 & 210 & 1.8 \\
\multirow{2}{*}{ Programme 1 } & Inner plate & CFRP & 40 & 4 \\
& Outer plate & CFRP & 40 & 4 \\
Programme 2 & Inner plate & DC590 & 210 & 1.6 \\
& Outer plate & CFRP & 40 & 4 \\
Programme 3 & Inner plate & CFRP & 40 & 4 \\
& Outer plate & DC590 & 210 & 1.8 \\
\hline
\end{tabular}


Option 3 replaces the inner plate of the B-pillar with equal rigidity. The inner thickness of the CFRP plate is $4 \mathrm{~mm}$, the thickness of each layer of the CFRP is $0.25 \mathrm{~mm}$, and the inner and outer plates are laid 16 layers, and the layers are laid in the manner of $0^{\circ} / 45^{\circ} / 90^{\circ} /-45^{\circ}$. The outer plate is of original metal structure and is rigidly connected.

\subsection{Static Analysis Results Comparison}

The same static analysis conditions as the basic model were adopted, and the load and constraints were consistent. The comparison of the analysis results is shown in Table 2. It can be seen from Figures 6-8 that compared with the basic

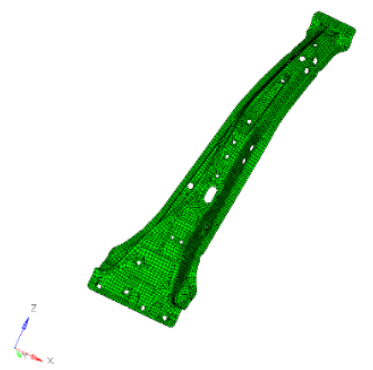

(a)

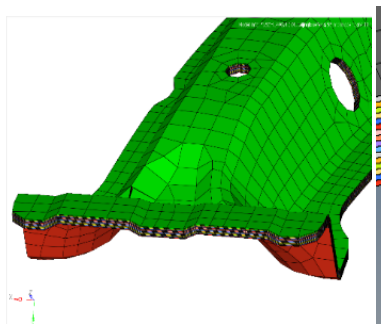

(b)



(c)

Figure 5. B-pillar outer layer. (a) B column outer panel; (b) Ply display; (c) Local layer display.

Table 2. Analysis of the results of three schemes for B column.

\begin{tabular}{llll}
\hline & quality $/ \mathrm{kg}$ & deformation $/ \mathrm{mm}$ & stress /Mpa \\
Basic model & 15.72 & 0.704 & 102 \\
Programme 1 & 11.59 & 0.591 & 34.5 \\
Programme 2 & 12.88 & 0.525 & 31.4 \\
Programme 3 & 14,44 & 0.286 & 88.4 \\
\hline
\end{tabular}

Contour Plot Displacement(Mag) Analysis system -7.038E-01

$-6.256 \mathrm{E}-01$

-5.474E-01

-4.692E-01

$-3.910 \mathrm{E}-01$

$-3.128 E-01$

$-2.346 \mathrm{E}-01$

$-1.564 \mathrm{E}-01$

$-7.820 \mathrm{E}-02$

$-0.000 E+00$

No result

Max $=7.038 \mathrm{E}-01$

Grids 9706

Min $=0.000 E+00$

Grids 11153

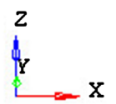

1: Model Contour Plot

1: Model Subcase 1 (loadstep1) : Static Analysis : Frame 25 Element Stresses

Element Stresses (2D \& 3D)(vonMises, Sabtase 1 (loadstep1) : Static Analysis : Frame 25



$-1.023 \mathrm{E}+02$
$-9.095 \mathrm{E}+01$
$-7.958 \mathrm{E}+01$
$-6.822 \mathrm{E}+01$
$-5.685 \mathrm{E}+01$
$-4.548 \mathrm{E}+01$
$-3.411 \mathrm{E}+01$
$-2.274 \mathrm{E}+01$
$-1.137 \mathrm{E}+01$
$1.104 \mathrm{E}-12$
No result
Max $=1.023 \mathrm{E}+02$
$2 \mathrm{D} 5152$
Min $=1.104 \mathrm{E}-12$
$2 \mathrm{D} 322$

(a)

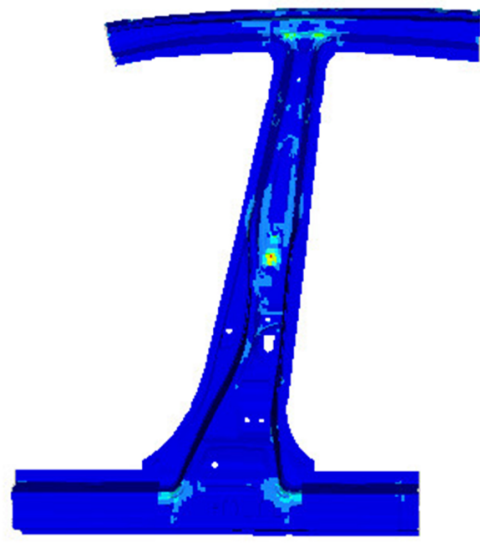

(b)

Figure 6. Scheme 1 displacement stress cloud map. (a) Deformation cloud; (b) Stress cloud. 


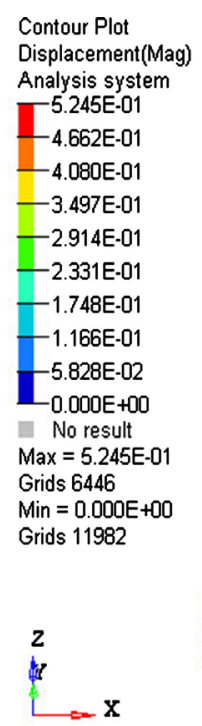

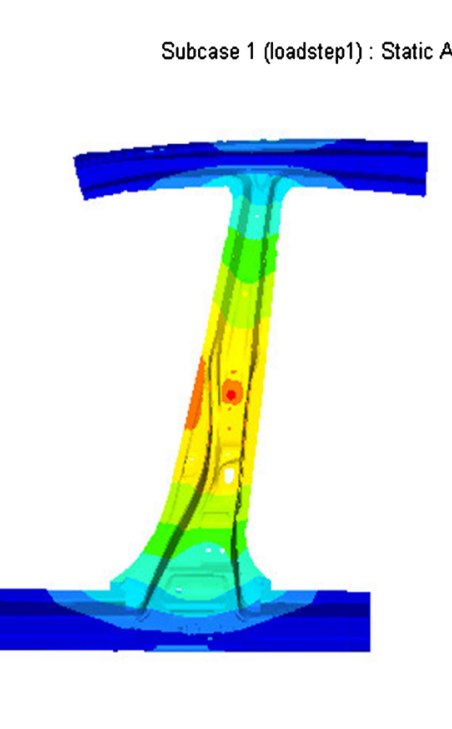

(a)
1: Model Contour Plot 1: Model Element Stresses (2D \& 3D)(vonMises, 乌laðdase 1 (loadstep1) : Static Analysis : Frame 25 Analysis system

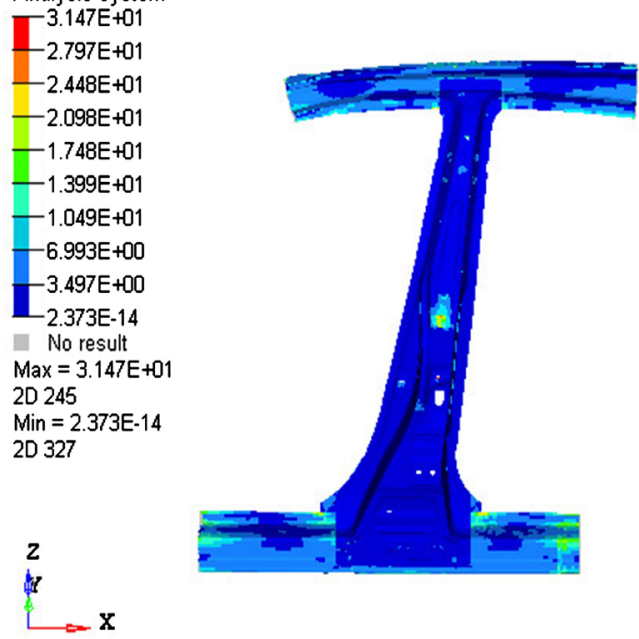

(b)

Figure 7. Scheme 2 displacement stress cloud map. (a) Deformation cloud; (b) Stress cloud.

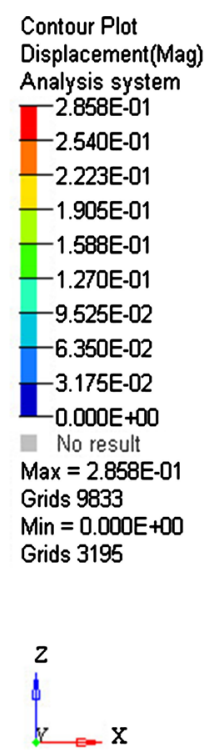

Displacement(Mag)

Analysis system

-2.858E-01

$-2.540 \mathrm{E}-01$

-2.223E-01

$-1.905 \mathrm{E}-01$

$-1.588 \mathrm{E}-01$

1.270E-01

.350E-02

$3.175 \mathrm{E}-02$

1. No result

Max = 2.858E-01

Grids 9833

Min $=0.000 E+0$

Grids 3195

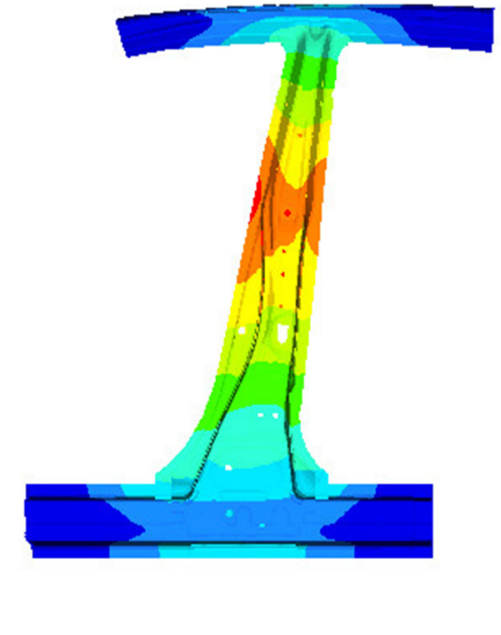

(a)
1: Model Contour Plot

1: Model Subcase 1 (loadstep1) : Static Analysis : Frame 25 Analysis system Analysis system

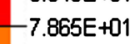

$-6.882 E+01$

$-5.899 \mathrm{E}+01$

$-4.916 \mathrm{E}+01$

$-3.932 \mathrm{E}+01$

$-2.949 E+01$

$-1.966 \mathrm{E}+01$

$-9.831 E+00$

-1.531E-13

- No result

$\operatorname{Max}=8.848 \mathrm{E}+01$

2D 5152

$\operatorname{Min}=1.531 \mathrm{E}-13$

2D 231
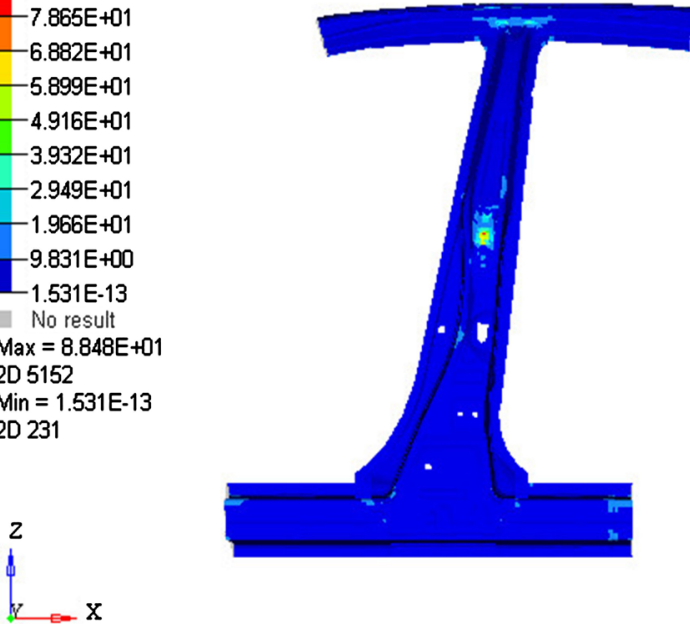

(b)

Figure 8. Scheme 3 displacement stress cloud map. (a) Deformation cloud; (b) Stress cloud.

model, the deformation of the B-pillar of the scheme 1 is reduced by $16 \%$, the deformation of the B-pillar of the scheme 2 is decreased by $25 \%$, the maximum stress position of the overall structure of the B-pillar is the same, and the maximum stress value of the scheme 1 and the scheme 2 is the same. Greatly reduced.

Through the design analysis and comparison of the three schemes, the replacement of the composite material inside and outside the B-pillar, the small stress value of the deformation of the B-pillar is small, and the weight reduction effect is better. Therefore, in the first option, the replacement study of the composite material of the B-pillar is carried out. The following is a study on the collision of the composite B-pillar with the original high-strength steel. 


\section{Analysis of Collision Performance of Automobile B-Pillar}

The analysis of the impact performance of the B-pillar of the car mainly considers the working conditions of the side impact [10]. The column setting in the reference establishes a cylinder with a radius of $100 \mathrm{~mm}$ and sets it as a rigid body; the four ends of the B-column constrain all translational degrees of freedom and rotational degrees of freedom. The rigid cylinder has a mass of $600 \mathrm{~kg}$ and strikes the middle of the B-pillar at an initial velocity of $2 \mathrm{~m} / \mathrm{s}$ along the Y-direction. The B-pillar collision analysis model is shown in Figure 9. In the side impact safety analysis, five reference points A1 to A5 are established, and the safety performance of the B-pillar structure is evaluated by using indicators such as side intrusion amount and intrusion speed.

By analyzing the change between the energy of the collision of the metal $\mathrm{B}$ column in Figure 10, it can be seen that the whole collision process is conserved, the total energy is $1200 \mathrm{~J}$, the hourglass energy is $48.5 \mathrm{~J}$, less than $5 \%$ of the total energy, less than $10 \%$. The overall trend indicates that the entire B-pillar collision is conserved, verifying the accuracy of the finite element collision model. Figure 11 is a stress cloud diagram of a metal B-column in the event of a collision. The peak of the intrusion is the maximum of $31.87 \mathrm{~mm}$ at the selected five reference points, and the peak of the intrusion velocity is $1108.2 \mathrm{~m} / \mathrm{s}$.

Taking the same collision analysis conditions as the basic model, the comparison of the intrusion amount and the intrusion velocity on the B-pillar is shown in Figure 12 and Figure 13, respectively, showing that the metal material and the carbon fiber composite are at the same three reference nodes. The displacement time curve can be seen from the comparison. The displacement of the B-pillar of the metal material is larger than the displacement of the B-pillar of the composite material, that is, the deformation of the B-pillar of the metallic material in the $y$ direction is larger than that of the composite material B. From



Figure 9. B-pillar collision analysis model. 


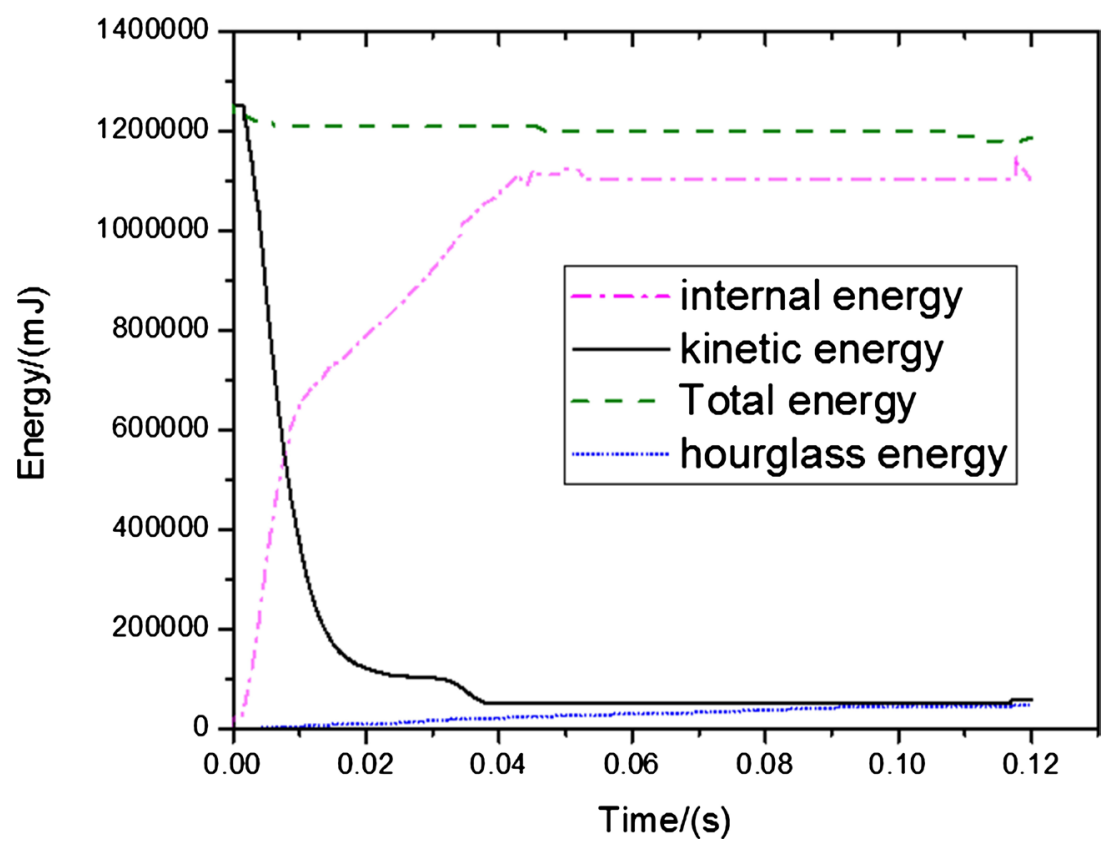

Figure 10. Energy curve.

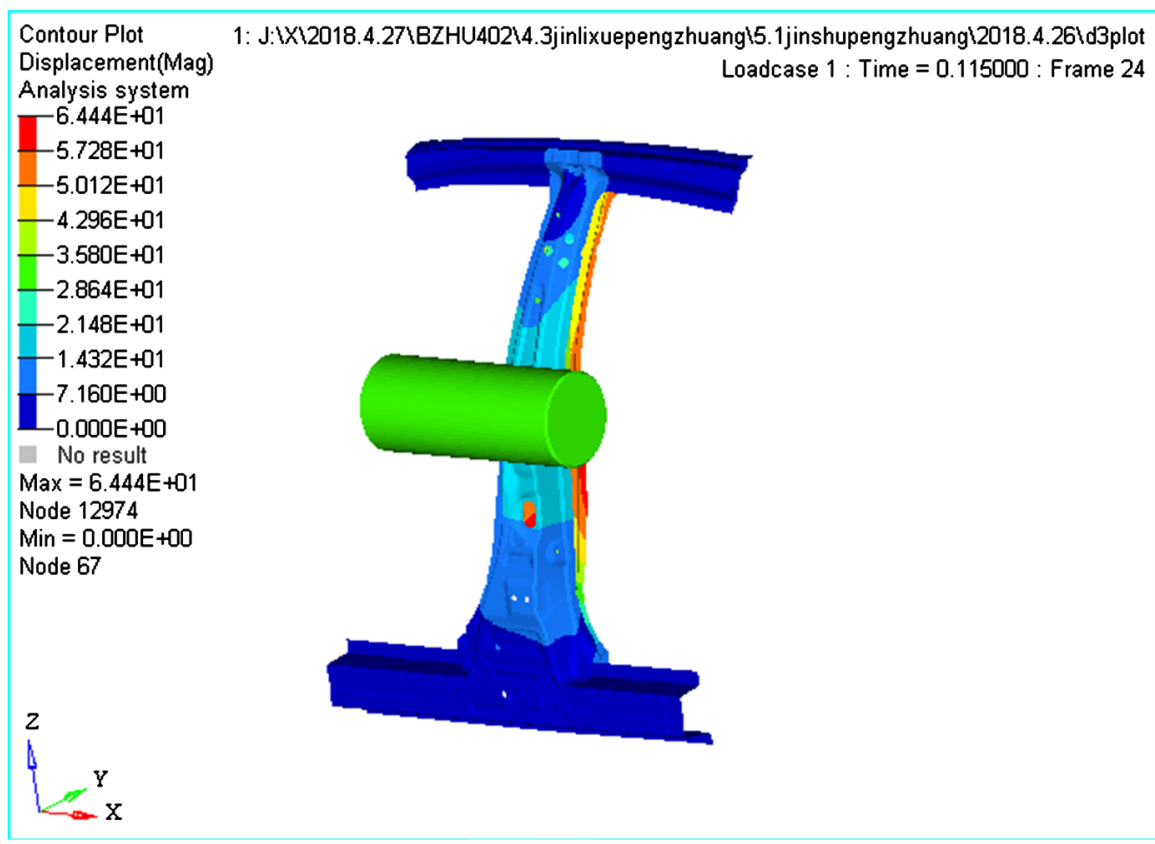

Figure 11. B-column collision cloud.

Figure 12, the intrusion amount of the three reference points output of A2, A3, and A4 is selected, and the displacement of the B-bar of the metal material increases first and then decreases with time, and the peak value of the intrusion amount of the three reference points is $45.60 \mathrm{~mm}$ in the middle of A3; It can be seen from 13 that the displacement of the three test points of the composite material B column increases first and then decreases with time. The maximum peak of the three reference points is $32.87 \mathrm{~mm}$ in the middle of A3. It can be visually seen that the deformation of the composite material is smaller than that of the metal material. 


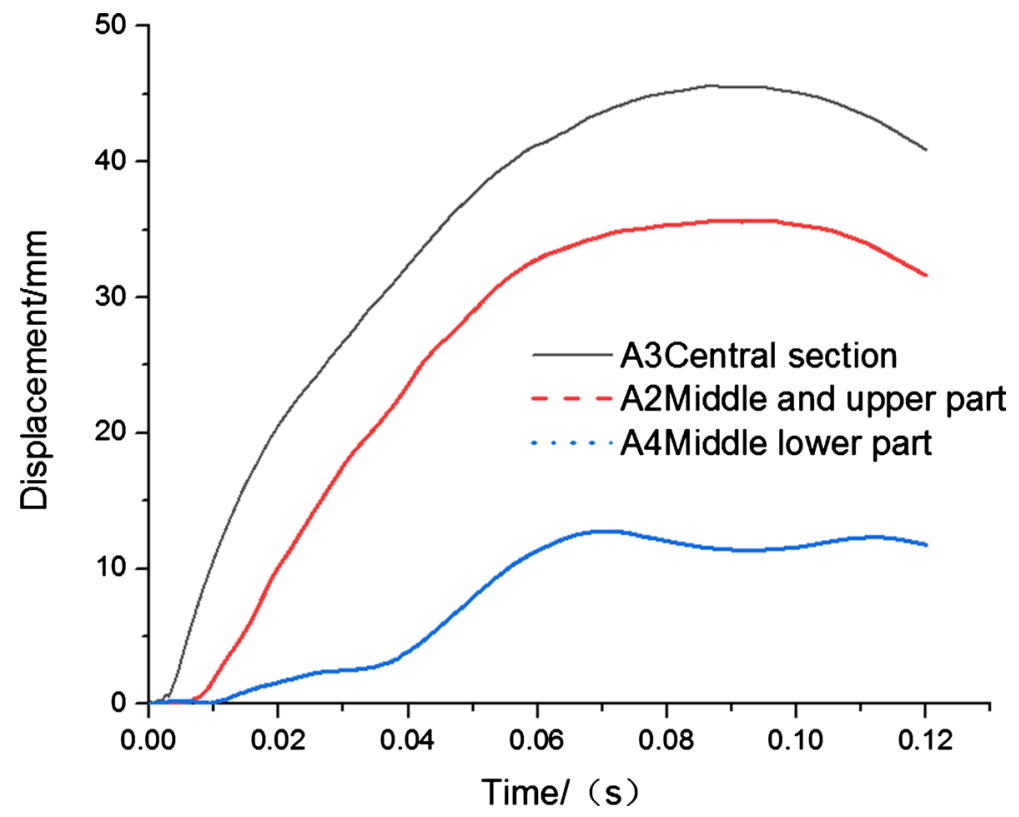

Figure 12. Metal material B-column displacement-time diagram.

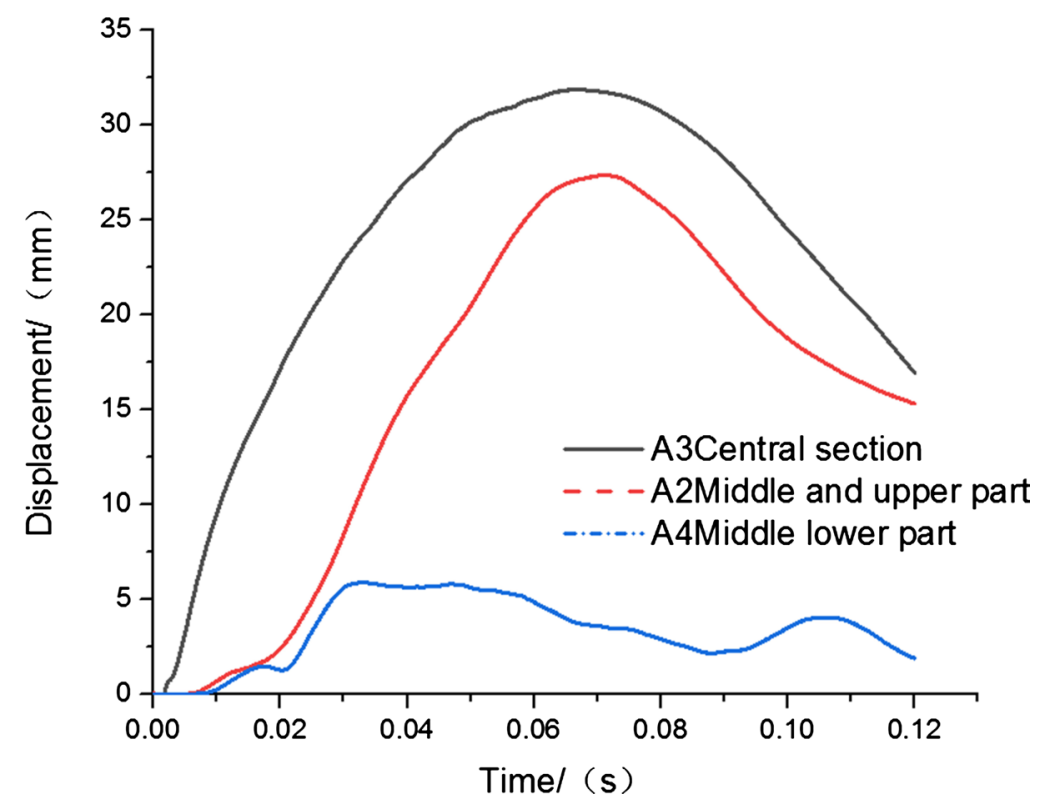

Figure 13. Composite B-pillar displacement-time diagram.

\section{Conclusion}

In this paper, the lightweight design of B-pillars was carried out, CFRP carbon fiber composites were selected instead, and the thickness of B-pillars was designed according to the principle of equal stiffness. Three lightweight schemes were designed. The finite element model is established, and the static and collision analysis of the B-pillar is carried out. It is known from the simulation results that: while ensuring the crashworthiness of the B-pillar, the B-pillar improves the crashworthiness, and the quality of the CFRP material is reduced by $26 \%$. The advantages of lightweight and comprehensive performance are ob- 
vious. CFRP material is superior to the original high-strength steel material and is a lightweight composite material with great reference value.

\section{Conflicts of Interest}

The authors declare no conflicts of interest regarding the publication of this paper.

\section{References}

[1] Guo, S.W., Yin, W.Y., Bu, X.B., et al. (2017) The Side Impact Safety Performance of an Electric Vehicle Is Improved by. Tianjin Science and Technology, 44, 64-66.

[2] Chen, X.P., Hu, X., Song, C., et al. (2016) Simulation and Experimental Study on Hydraulic Forming B Column of Super High Strength Steel QP980. Precision Forming Engineering, 8.

[3] Gui, K.H., Tang, C.G., Lu, H.G., et al. (2017) Research on Impact Performance of Hot Stamping B Column. Automotive Practical Technology, 8, 127-129.

[4] Wu, F.H. (2017) Structural Design and Optimization of Carbon Fiber Composite Engine Hood. Jilin University, Jilin.

[5] Lightweight Design and Optimization of Lee Bei Fiber Reinforced Composite Bumper. Hunan University, Changsha.

[6] Zhang, Z.C., Zhu, P., Feng, Q., et al. (2015) Study on Optimum Design of Carbon Fiber Composite Car Wing Plate. Journal of Automobile Engineering, 5, 367-374.

[7] Peng (2011) Study on Composite Structure Design and Energy Absorption Characteristics of Automobile Front Rails. Harbin Institute of Technology, Harbin.

[8] Yang, Y.W., Tian, Y.L., Liu, X.X., et al. (2017) Development of Car Body Structural Components of Integrated Carbon Fiber Composites Pure Electric Vehicle. FRP/ Composite, 7, 65-69.

[9] Xue, J. (2013) Simplified Structural Parameter Analysis of Carbon Fiber Reinforced Polymer B Column.

[10] Gao, X., Fan, T.Q., Zhao, Q.J., et al. (2014) Applied Research of High Strength Steel B Column Parts Based on Finite Element Analysis. Automobile Technology and Materials, 9, 10-14. 